BULL. AUSTRAL, MATH. SOC.

VOL. $33(1986), 477-479$.

\title{
INTEGRABLE DOUBLE KERR-SCHILD SPACES
}

\author{
MARK S, HICKMAN
}

Exact solutions of the vacuum Einstein field equations have been sought for the last seventy years. In the 1960's a class of solutions, known as Kerr-Schild solutions, were investigated. This class includes solutions of great importance. For example the Schwarschild and Kerr metrics are both members of this class. Unfortunately this class has proven to be restrictive. This thesis is mainly concerned with a generalization of this class, known as integrable double Kerr-Schild solutions. Solutions of this class possess a totally null foliation of codimension 2 and can be written in the form

$$
g=g_{0}+P 1 \otimes 1+2 R 1 \otimes m+Q m \otimes m
$$

where $g_{0}$ is a flat space metric, 1 and $m$ are any basis for the foliation and $P, Q$ and $R$ are complex valued functions. The Lorentz signature of physical spacetimes necessitates integrable double KerrSchild solutions to be complex solutions of the Einstein field equations. Vacuum solutions of this class include, for example, the analytic continuation of any real algebraically degenerate vacuum spacetime.

The necessary modifications of standard relativity theory are given to allow the discussion of complex spaces. In particular, the wel1-known Newman-Penrose formalism [1] and the 2-component spinor formalism [2] are

Received 29 August 1985. Thesis submitted to Monash University, February 1985. Degree approved July 1985. Supervisor:

Dr. C.B.G. McIntosh.

Copyright Clearance Centre, Inc. Serial-fee code: 0004-9727/86 $\$ A 2.00+0.00$. 
adapted for complex spaces. Some of the novel features of complex spaces are examined. In particular, a classification scheme based upon the properties of complex wave surfaces of a null direction is given. The most important subcase of this scheme is that of the integrable double Kerrschild spaces. The wave surfaces give rise to a particularly useful tetrad for studying these spaces.

The potentialization of Plebañski and Robinson $[3,4]$ of vacuum integrable double Kerr-Schild spaces is obtained by a novel method. Seven of the ten vacuum field equations are satisfied identically with the remaining three equations reduced to a single equation for the potential. The potentialization is then specialized to the Petrov type $N \otimes N$ case (that is both the self-dual and anti-self-dual Weyl tensor are null). Several subcases are integrated yielding the complex analogues of the Robinson-Trautman, Kundt and $p p$ wave metrics plus two solutions which do not possess real analogues (of Lorentz signature).

The canonical tetrad for integrable double Kerr-Schild spaces is exploited to give an account of the Cohen and Kegeles theory of spin $1 / 2$ and 1 fields $[5,6]$ in conformally integrable double Kerr-Schild backgrounds. The canonical tetrad allows the calculations to be completed in a more direct manner and to obtain the results in a covariant form. The Cohen and Kegeles treatment of gravitational perturbations of algebraically degenerate vacuum spacetimes $[6,7]$ is shown to be a first order perturbation of the potential of Plebañski and Robinson. This potentialization is exploited to give an account of a higher order perturbation scheme for vacuum integrable double Kerr-Schild backgrounds.

\section{References}

[1] E.T. Newman and R. Penrose, "An approach to gravitational radiation by the method of spin coefficients", J. Math. Phys. 3 (1962), 556-578.

[2] R. Penrose, Stmucture of spacetime, Battelle Rencontre, (1967) Lectures in Mathematics and Physics, ed. C.M. Dewitt and J.A. Wheeler, Benjamin, New York, 1968

[3] J.F. Plebañski and I. Robinson, "Left degenerate vacuum metrics", Phys. Rev. Lett. 37 (1976), 493-495. 
[4] J.F. Plebañski and I. Robinson, The complex vacum metrics with minimally degenerate conformal curvature (Asymptotic structure of spacetime, ed. E.P. Esposito and L. Witten, Plenum Press, New York, 1977).

[5] J.M. Cohen and L.S. Kegeles, "Electromagnetic fields in curved spaces: A constructive procedure", Phys. Lett. A47 (1974), 261-262.

[6] L.S. Kegeles and J.M. Cohen, "Constructive procedure for perturbations of spacetime", Phys. Rev. D19 (1979), 1641-1664.

[7] J.M. Cohen and L.S. Kegeles, "Spacetime perturbations", Phys. Lett. A54 (1975), 5-7.

Department of Mathematics,

Monash University,

Clayton, Vic. 3168,

Australia. 